\title{
Noninvasive measurement of respiratory muscle performance after exhaustive endurance exercise
}

\author{
C. Perret, R. Pfeiffer, U. Boutellier, H.M. Wey, C.M. Spengler
}

\begin{abstract}
Noninvasive measurement of respiratory muscle performance after exhaustive endurance exercise. C. Perret, R. Pfeiffer, U. Boutellier, H.M. Wey, C.M. Spengler. (C)ERS Journals Ltd 1999.

ABSTRACT: The use of noninvasive techniques to measure respiratory muscle performance after different types of endurance exercise has not been entirely successful, as the results have not consistently indicated diminished performance for similar types of exercise. The aim of the present study was 1) to compare different, noninvasive methods to assess respiratory muscle performance before and after an exhaustive cycling endurance test (which has previously been shown to induce diaphragmatic fatigue) and 2) to determine which of the tests best reflect published results of measurements of diaphragmatic fatigue.

Twelve healthy subjects participated in the study and performed three different test series in a random order on three different days. These tests were performed before, and 5, 40 and 75 min after an exhausting task (a cycling endurance run at $85 \%$ of maximal oxygen uptake $\left.\left(V^{\prime} \mathrm{O}_{2}, \mathrm{max}\right)\right)$. The tests of the three test series were 1) breathing against a constant inspiratory resistance to task failure, 2) determination of 12 -min sustained ventilatory capacity, and 3) spirometric and maximal inspiratory and expiratory mouth pressure measurements.

The only measurement that was affected by exhaustive cycling was the time to task failure breathing against inspiratory resistance. It was significantly reduced from (mean $\pm \mathrm{SD}$ ) $364 \pm 88 \mathrm{~s}$ before exercise to $219 \pm 122 \mathrm{~s}$ at $5 \mathrm{~min}$ after cessation of exercise.

It is concluded that the constant-load resistive breathing test to task failure is the only noninvasive respiratory muscle performance test evaluated in this study which shows a decrease in respiratory muscle performance after exhaustive endurance exercise.
\end{abstract}

Eur Respir J 1999; 14: 264-269. \author{
Zurich, Switzerland.

\section{Correspondence: C. Perret} \\ Sportphysiologie \\ Winterthurerstr. 190 \\ CH-8057 Zürich \\ Switzerland \\ Fax: 4116356814
}

Exercise Physiology, Swiss Federal Institute of Technology, and University of Zurich, Winterthurerstrasse 190, CH-8057

Keywords: Endurance exercise inspiratory resistive load respiratory muscle fatigue

Received: July 71998

Accepted after revision March 71999

Financial support was provided by the Swiss National Science Foundation (grant no. 32-43391-95)
A number of different methods have been suggested for noninvasive measurement of global respiratory muscle fatigue [1]. To assess fatigue of the diaphragm specifically, supramaximal phrenic nerve stimulation, i.e. measurement of twitch transdiaphragmatic pressure $(P \mathrm{di}, \mathrm{tw})$, is currently regarded as being the most objective measure of diaphragmatic fatigue. Exercise to exhaustion at $80 \%$ maximal working capacity $\left(W_{\max }\right)[2]$ as well as at $85-$ $95 \%$ maximal oxygen uptake $\left(V^{\prime} \mathrm{O}_{2}, \max \right)[3,4]$ induces diaphragmatic fatigue as shown by a reduced $P$ di,tw after exercise. While a decrease in $P$ di,tw is an unequivocal measure of diaphragmatic fatigue, in field studies less invasive measurements are needed. A number of commonly used, noninvasive methods have been examined, but the results regarding reduced respiratory muscle performance after different types of endurance exercise are conflicting.

One way of determining whether respiratory muscle performance is reduced after exercise is by examining the ability of respiratory muscles to generate maximal force through measuring maximal inspiratory $(P \mathrm{I}, \mathrm{max})$ and expiratory $(P E, \max )$ mouth pressures. One such study showed a significant reduction of $P \mathrm{I}$,max and $P \mathrm{E}$,max after a marathon run [5], others found reductions only in $P \mathrm{I}$,max while $P$ E,max remained unchanged after a triathlon [6], after a marathon [7] and after an incremental, multi-stage shuttle run to volitional fatigue [8]. No reduction in either $P$ I,max or $P$ E,max was found after a $24 \mathrm{~h}$ ultramarathon [9]. Another means to assess respiratory muscle performance is to carry out short or long term respiratory endurance tasks, e.g. maximal voluntary ventilation (MVV) or sustained ventilatory capacity (SVC), or to investigate changes of maximal flow tasks, e.g. forced expiratory volume in one second (FEV1) or forced vital capacity (FVC). Contradictory results have also been found using these variables. In some subjects, MVV was reduced after running a marathon [5], while it did not change after similar endurance competitions in other studies $[6,7,9]$. FVC was reduced in subjects after running 8 to $100 \mathrm{~km}$ [10-13] and after performing a triathlon [6]. Other studies did not confirm these results $[5,7,14,15]$. FEV1 and mean expiratory flow (MEF) reductions were reported in some of the above mentioned studies $[6,10,14]$ while they remained unchanged in others $[5,7,11,13,15]$. Finally, KER and SCHULTZ [16] showed that inspiratory muscle endurance time, as measured by sustained inspiratory mouth pressures at a given target pressure and a given duty cycle, was still impaired 3 days after an uotramarathon.

The above reported, noninvasive measurements of respiratory muscle performance were applied to a wide range 
of endurance exercises of different types and duration. This might explain the contradictions in the results of the different studies. Alternately, these noninvasive measurements (force, endurance or maximal flow tasks) may not all be equally well suited to detecting reduced respiratory muscle performance occurring after intensive exercise. Therefore, the aim of the present study was to compare different, noninvasive tests of respiratory muscle performance (constant-load resistive breathing to task failure, SVC, MVV, vital capacity (VC), FVC, FEV1, peak expiratory flow (PEF), $P \mathrm{I}$,max and $P \mathrm{E}, \max )$ to assess respiratory muscle performance before and after cycling to exhaustion at a constant load corresponding to $85 \% V^{\prime} \mathrm{O}_{2}$, max, an intensity previously shown to induce diaphragmatic fatigue by measurement of $P$ di,tw $[3,4]$.

\section{Material and methods}

\section{Study subjects}

Twelve healthy, nonsmoking subjects (4 females, 8 males) participated in the study. Their average age was (mean \pm SD) $28 \pm 7$ yrs, height $177 \pm 10 \mathrm{~cm}$ and weight $70 \pm 10$ $\mathrm{kg}$. The study was performed in accordance with the ethical standards of the Helsinki Declaration for experimentation on human subjects. Subjects were familiarized with the experimental procedures and informed consent was obtained.

\section{Study design}

Subjects carried out respiratory performance tests before, and 5, 40 and $75 \mathrm{~min}$ after a cycling endurance test at $85 \% \mathrm{~V}^{\prime} \mathrm{O}_{2}$, max continued until exhaustion. The tests consisted of 1) breathing against a constant inspiratory resistance to task failure, 2) determination of the 12-min sustained ventilatory capacity or 3 ) spirometric measurements and determination of maximal inspiratory and expiratory mouth pressures. The test groups $(1,2$, or 3$)$ were performed on three different days in a random order.

\section{Preliminary tests}

On three different days, the following preliminary tests were performed.

Incremental breathing test. Subjects began by breathing against an inspiratory resistive load at a pressure corresponding to $60 \% \mathrm{PI}$,max (determined at residual volume). Expiration was unloaded and breathing frequency $(f \mathrm{R})$ was set at 18 breaths. $\min ^{-1}$ and paced by a metronome. Every $3 \mathrm{~min}$ the resistive load was increased by $5 \%$ $P$ I,max. The test continued until the subjects were no longer able to overcome the load. The inspiratory pressure $(P \mathrm{I})$ of the last step which the subjects were able to sustain for $3 \mathrm{~min}$, was selected as the target pressure for the constant-load resistive breathing test (see below).

Two consecutive constant-load resistive breathing tests. On a different day, subjects performed two consecutive constant-load resistive breathing tests at a constant load corresponding to (mean \pm SD) $79 \pm 9 \%$ PI,max. The two tests were separated by a 15 min rest period. During each test, the subjects matched their inspiratory pressure to a pressure waveform (previously determined to be comfortable) displayed to them on an oscilloscope at an $f \mathrm{R}$ of $18 \mathrm{breaths} \cdot \mathrm{min}^{-1}$. The time to task failure (ttask failure) was defined as the time when the subjects were no longer able to achieve the target pressure. During this test, subjects were asked to rate their respiratory exertion on a modified Borg scale every minute.

Incremental cycling test. An incremental cycling test was performed to exhaustion in order to determine $V^{\prime} \mathrm{O}_{2}$,max. The work load on the cycle ergometer was set at $100 \mathrm{~W}$ and was increased by $30 \mathrm{~W}$ every $2 \mathrm{~min}$. Pedalling frequency was maintained at a constant level throughout the test. Subjects chose their preferred frequency at the beginning of the test.

\section{Main experiments}

The following tests were performed at least once before the actual testing, and the main experiments consisted of the three test series, each one performed on different days separated by at least $48 \mathrm{~h}$. Each test series began (t0) with one of the three respiratory muscle performance tests (see below). Fifteen minutes after the end of the respiratory muscle performance test, subjects started cycling at $85 \%$ $V^{\prime} \mathrm{O}_{2}$, max and continued until exhaustion. At $5 \mathrm{~min}(t 5), 40$ $\min (t 40)$ and 75 min (t75) after the subjects stopped cycling, the respiratory muscle performance tests were repeated. These respiratory tests before and after cycling consisted of 1) measuring the time that the subjects could breathe against a constant inspiratory resistance (inspiratory resistance was individually determined, see above; average mean $\pm \mathrm{SD}) 79 \pm 9 \% P \mathrm{I}$,max, corresponding to $-133 \pm$ $25 \mathrm{cmH}_{2} \mathrm{O}$ ) (Task failure-series), 2) determining the 12min SVC (SVC-series) or 3) measurement of spirometric variables (VC, FVC, FEV1, PEF, MVV in 15 seconds (MVV15), $P$ I,max and $P$ E,max (Spiro-series). These three test series were performed in a randomized order. During the Task failure-series, subjects were asked to rate their perceived respiratory exertion every minute on a modified Borg scale. Before and after every respiratory muscle performance test-series, a $20 \mu \mathrm{L}$ blood sample was taken from an earlobe to analyse for blood lactate concentration. Cycling endurance tests were started at $100 \mathrm{~W}$, and the workload was increased in three equal increments of 2 min duration to reach $85 \% V^{\prime} \mathrm{O}_{2}$, max within $6 \mathrm{~min}$. The exact levels achieved were $78 \pm 3 \%$ of $W_{\max }(245 \pm 50 \mathrm{~W})$ corresponding to $86.9 \pm 4.6 \% \mathrm{~V}^{\prime} \mathrm{O}_{2}$, max for the Task failure-

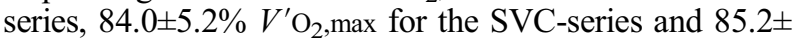
$5.6 \% V^{\prime} \mathrm{O}_{2}$, max for the Spiro-series.

\section{Equipment}

Spirometric variables (VC, FVC, FEV1, PEF, MVV15), SVC as well as ventilatory and gas exchange variables during cycling were determined with an ergo-spirometric device, (Oxycon Beta; Mijnhardt, Bunnik, Netherlands) using a turbine flow meter for volume measurements, a paramagnetic analyser for $\mathrm{O}_{2}$, and an infrared absorption 
analyser for $\mathrm{CO}_{2}$-measurements. For SVC-measurements, subjects were instructed to breathe maximally for a period of $12 \mathrm{~min}$. The average minute ventilation for the entire 12 min was then calculated. To maintain isocapnia during the 12-min SVC-measurement, a partial rebreathing device was used. It consisted of a latex bag connected to a tube with inlet and outlet valves. The size of the bag was adjusted to be $50-60 \%$ of the subjects' VC. Subjects were instructed to fill and empty the bag completely while sufficient inspiratory and expiratory flow through the valves was permitted to ensure isocapnia and full oxygen saturation. Minute ventilation was mainly varied by changing $f \mathrm{R}$. In a preliminary session, subjects were trained to use this device. During the tests, the settings kept end tidal $\mathrm{CO}_{2}$ in the normal range (SVC at t0: $5.4 \pm 0.8 \mathrm{kPa}(40.9 \pm 6.0$ $\mathrm{mmHg}) ; \mathrm{SVC}$ at $t 5: 5.1 \pm 0.7 \mathrm{kPa}(38.0 \pm 4.9 \mathrm{mmHg}) ; \mathrm{SVC}$ at $t 40: 5.1 \pm 0.7 \mathrm{kPa}(38.0 \pm 4.9 \mathrm{mmHg}) ; \mathrm{SVC}$ at $t 75: 5.0 \pm 0.7$ $\mathrm{kPa}(37.9 \pm 5.4 \mathrm{mmHg}$ ). Measurement of $P$ I,max (at residual volume) and $P$ E,max (at total lung capacity) as well as constant-load resistive breathing were performed on a selfdeveloped device (Tecuria, Chur, Switzerland). This device consists of a mouthpiece connected to a tube system including a flow sensor (163PC01D75; Honeywell Inc., Phoenix, AZ, USA) and a pressure sensor (143C05PCB; Sensym Inc., Milpitas, CA, USA). The tube system extends to two electronically controlled valves (inspiratory and expiratory). Breathing resistance increases proportionally to the voltage applied to the valves. The mouth pressure generated was displayed online on the screen of an oscilloscope on which the target pressure trace was outlined. Respiratory frequency and duty cycle were paced by the sweep time of the cathode beam. Cycling tests were performed on an electronically breaked cycle ergometer (Ergometrics 800; Ergoline, Bitz, Germany). Blood samples were analysed enzymatically for blood lactate concentration by an ESAT 6661 analyser (Eppendorf, Hamburg, Germany). Subjects rated their perceived respiratory exertion on a modified Borg scale (0-10).

\section{Analysis}

For comparison of ventilatory variables during the constant-load resistive breathing tests, average values over the entire test-time were calculated. Ventilatory variables during the cycling tests were averaged during the constantload period, with the first and last $1.5 \mathrm{~min}$ of this period being discarded to avoid the inclusion of any hyperventilation which may have occurred shortly before the end of exercise. To test for significant differences between the respiratory muscle performance tests at the four different times of one test-series, the nonparametric Friedman's analysis of variance was used. If a significance was found a Wilcoxon and Wilcox comparison was used to locate the significant differences. The same statistics were used to test for significant differences between variables of different respiratory muscle performance test-series at equal timepoints before or after exercise as well as between variables of the three cycling endurance tests. Values of the two preliminary consecutive resistive breathing tests were compared using the paired Wilcoxon's signed rank test. Results are given as mean \pm SD. Values were considered to be significantly different if $\mathrm{p}<0.05$.

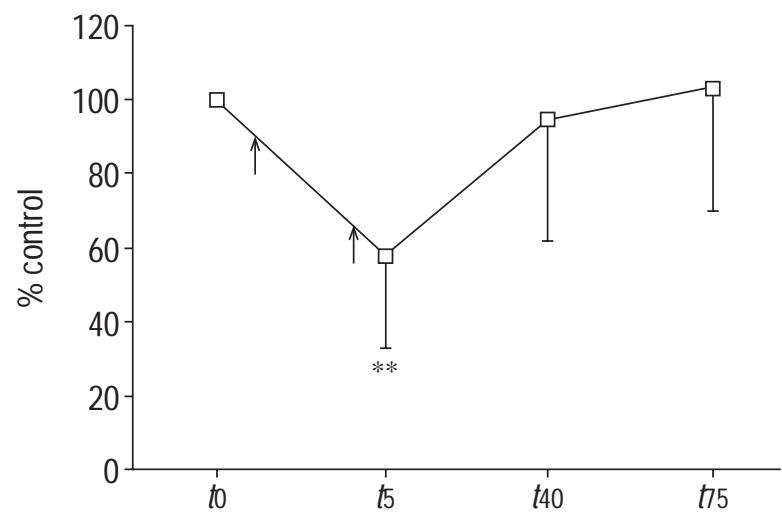

Fig. 1. - Time to task failure (ttask failure) during the constant-load resistive breathing test before $(t 0)$ and $5(t 5), 40(t 40)$, and $75 \mathrm{~min}(t 75)$ after a cycling endurance test (CET) in 12 subjects. Values are given as percentage of control values $(t 0) . \square$ : $t$ task failure; $* *: \mathrm{p}<0.01$; arrows indicate the start and end of CET.

\section{Results}

Time to task failure of the constant-load resistive breathing test was significantly reduced at $5 \mathrm{~min}$ after cessation of exercise (t5) compared to values before the exhaustive exercise (fig. 1). All other respiratory muscle performance tests were unaffected by prior exhaustive cycling (table 1). Average minute ventilation and breathing pattern were not significantly different during the constant-load resistive breathing tests at $t 5, t 40$ and $t 75$ from to (table 2). Blood lactate concentrations before respiratory performance tests did not differ significantly between test series at $t 0(1.5 \pm 0.6$ (Task failure-series), $1.2 \pm 0.4$ (SVC-series), $1.3 \pm 0.4 \mathrm{mmol} \cdot \mathrm{L}^{-1}$ (Spiro-series) $), t 5$ $\left(7.7 \pm 1.7,7.4 \pm 1.8,7.0 \pm 2.0 \mathrm{mmol} \cdot \mathrm{L}^{-1}\right), t 40(2.0 \pm 0.6,1.9 \pm$ $\left.0.4,1.9 \pm 0.7 \mathrm{mmol} \cdot \mathrm{L}^{-1}\right)$ and $t 75(1.3 \pm 0.4,1.3 \pm 0.3,1.5 \pm 0.5$ $\left.\mathrm{mmol} \cdot \mathrm{L}^{-1}\right)$. During the three exhaustive cycling tests, average minute ventilation, breathing pattern and times to exhaustion did not differ significantly either (table 3 ).

Average times to task failure in the two preliminary, consecutive constant-load resistive breathing tests were

Table 1. - Data from respiratory muscle performance tests

\begin{tabular}{lcccc}
\hline & $t 0$ & $t 5$ & $t 40$ & $t 75$ \\
\hline$t$ task failure s & $364 \pm 88$ & $219 \pm 122 *$ & $346 \pm 135$ & $370 \pm 119$ \\
SVC L min $^{-1}$ & $141 \pm 22$ & $139 \pm 21$ & $136 \pm 17$ & $136 \pm 19$ \\
MVV15 & & & & \\
$\quad$ L min $^{-1}$ & $188 \pm 31$ & $190 \pm 33$ & $188 \pm 33$ & $183 \pm 34$ \\
VC L & $5.72 \pm 0.89$ & $5.74 \pm 0.93$ & $5.72 \pm 0.92$ & $5.78 \pm 0.92$ \\
FVC L & $5.58 \pm 0.84$ & $5.56 \pm 0.82$ & $5.49 \pm 0.77$ & $5.51 \pm 0.80$ \\
FEV1 L & $4.55 \pm 0.76$ & $4.60 \pm 0.73$ & $4.53 \pm 0.69$ & $4.49 \pm 0.71$ \\
PEF L $\cdot$ s $^{-1}$ & $9.9 \pm 2.1$ & $9.6 \pm 1.9$ & $9.7 \pm 2.1$ & $9.5 \pm 1.9$ \\
PI,max mbar & $-169 \pm 25$ & $-167 \pm 21$ & $-160 \pm 22$ & $-166 \pm 18$ \\
$P$ E,max mbar & $197 \pm 26$ & $191 \pm 38$ & $188 \pm 37$ & $189 \pm 36$ \\
\hline
\end{tabular}

Absolute values (mean $\pm \mathrm{SD})$ of time to task failure ( $t$ task failure), sustained ventilatory capacity (SVC), maximal voluntary ventilation in 15 seconds (MVV15), vital capacity (VC), forced vital capacity (FVC), forced expiratory volume in one second (FEV1), peak expiratory flow (PEF), maximal inspiratory and expiratory mouth pressures $(P \mathrm{I}, \max$ and $P \mathrm{E}, \max )$ before $(t 0)$ and 5 $(t 5), 40(t 40)$ and $75 \mathrm{~min}(t 75)$ after an exhaustive cycling endurance test $(\mathrm{n}=12){ }^{*}: \mathrm{p}<0.01 .1 \mathrm{mbar}=0.1 \mathrm{kPa}$. 
Table 2. - Data from the constant-load resistive breathing test

\begin{tabular}{lcccc}
\hline & $t 0$ & $t 5$ & $t 40$ & $t 75$ \\
\hline$V^{\prime} \mathrm{E} \mathrm{L} \cdot \mathrm{min}^{-1}$ & $15.0 \pm 2.4$ & $15.8 \pm 3.1$ & $14.1 \pm 2.1$ & $14.5 \pm 1.9$ \\
$t \mathrm{I} \mathrm{s}$ & $1.32 \pm 0.28$ & $1.30 \pm 0.28$ & $1.38 \pm 0.25$ & $1.30 \pm 0.27$ \\
$t \mathrm{E} \mathrm{S}$ & $1.92 \pm 0.26$ & $1.98 \pm 0.28$ & $1.91 \pm 0.24$ & $1.93 \pm 0.29$ \\
$V \mathrm{~T} / \mathrm{t} \mathrm{L} \mathrm{L} \cdot \mathrm{s}^{-1}$ & $0.64 \pm 0.17$ & $0.71 \pm 0.24$ & $0.56 \pm 0.14$ & $0.62 \pm 0.15$ \\
Respiratory exertion (first min) & $5.2 \pm 2.4$ & $6.1 \pm 2.4$ & $5.5 \pm 2.5$ & $5.5 \pm 2.5$ \\
Respiratory exertion (last min) & $9.3 \pm 1.1$ & $9.3 \pm 1.1$ & $9.3 \pm 1.2$ & $9.3 \pm 1.4$ \\
\hline
\end{tabular}

Absolute values (mean $\pm \mathrm{SD})$ of minute ventilation $\left(V^{\prime} \mathrm{E}\right)$, inspiratory time $(t \mathrm{I})$, expiratory time $(t \mathrm{E})$, inspiratory flow (tidal volume $\left.(V \mathrm{~T}) / t \mathrm{I}\right)$ and ratings of perceived respiratory exertion during the constant-load resistive breathing test (respiratory variables are given as mean values over the entire test; perceived exertion is given as values at the end of the first and last minute of the test) before (t0) and $5(t 5), 40$ $(t 40)$, and 75 min (t75) after an exhaustive cycling endurance test $(\mathrm{n}=12)$.

$390 \pm 141$ and $388 \pm 118$ s, respectively. They were not significantly different. Also, minute ventilation $\left(V^{\prime} \mathrm{E}: 15.1 \pm\right.$ 3.6, $14.5 \pm 2.6 \mathrm{~L} \cdot \mathrm{min}^{-1}$ ) and breathing pattern (inspiratory time $(t \mathrm{I}): 1.3 \pm 0.3,1.3 \pm 0.3 \mathrm{~s}$; expiratory time $(t \mathrm{E}): 2.0 \pm 0.2$, $1.9 \pm 0.2 \mathrm{~s}$; inspiratory flow (tidal volume $(V \mathrm{~T})) / t \mathrm{I}: 0.7 \pm 0.2$ $\left.0.6 \pm 0.2 \mathrm{~L} \cdot \mathrm{s}^{-1}\right)$ did not differ significantly.

\section{Discussion}

The only test to show a decrease in respiratory muscle performance after exhaustive endurance exercise was the constant-load resistive breathing test leading to task failure. Neither SVC, measures of lung function, i.e. VC, FVC, FEV1, PEF and MVV15, nor $P \mathrm{I}$,max and $P$ E, max were significantly reduced after cycling.

Possible factors other than any type of fatigue, which might have led to the reduced respiratory muscle performance during constant-load resistive breathing after exhaustive cycling need to be considered. In a subject-limited endurance test such as breathing to exhaustion against a resistance, subject's motivation is crucial. To avoid lack of motivation influencing the outcome of the study, only highly motivated subjects were chosen to participate. In fact, ratings of perceived respiratory exertion (table 2) which were similar at the end of all four constant-load resistive breathing tests, suggest that highly motivated subjects were successfully recruited and that they performed as hard as possible and to similar degrees of exertion at any time. In addition it is believed that, if motivation was a factor, reduced performance would also have occurred at t5 during the 12-min SVC-test as this test lasted longer than the constant-load resistive breathing test.

Alternatively, a change in minute ventilation and/or breathing pattern, as shown by CLANTON et al. [17], could have been responsible for a reduced endurance time

Table 3. - Data from three cycling endurance tests

\begin{tabular}{lccc}
\hline Variables & $\begin{array}{c}\text { Task failure- } \\
\text { series }\end{array}$ & $\begin{array}{c}\text { SVC- } \\
\text { series }\end{array}$ & $\begin{array}{c}\text { Spiro- } \\
\text { series }\end{array}$ \\
\hline$V^{\prime} \mathrm{E} \mathrm{L} \cdot \mathrm{min}^{-1}$ & $98.9 \pm 14.0$ & $98.9 \pm 13.2$ & $94.1 \pm 14.1$ \\
$t \mathrm{I} \mathrm{s}$ & $0.84 \pm 0.16$ & $0.82 \pm 0.11$ & $0.85 \pm 0.18$ \\
$t \mathrm{E} \mathrm{S}$ & $0.93 \pm 0.19$ & $0.88 \pm 0.15$ & $0.92 \pm 0.16$ \\
$V \mathrm{~T} / \mathrm{tI} \mathrm{L} \cdot \mathrm{s}^{-1}$ & $3.45 \pm 0.51$ & $3.38 \pm 0.44$ & $3.26 \pm 0.52$ \\
Time to exhaustion min & $25.7 \pm 6.6$ & $25.5 \pm 6.7$ & $26.0 \pm 9.8$
\end{tabular}

Absolute values (mean $\pm \mathrm{SD})$ of minute ventilation $\left(V^{\prime} \mathrm{E}\right)$, inspiratory time $(t \mathrm{I})$, expiratory time $(t \mathrm{E})$, inspiratory flow (ventilatory time $(V \mathrm{~T}) / t \mathrm{I})$, and time to exhaustion during the three cycling endurance tests (CET) in the Task failure-series, the SVC-series and the Spiro-series $(n=12)$. during constant-load resistive breathing at $t 5$ compared to to. However, no significant changes were observed in minute ventilation and breathing pattern (table 2) after cycling compared to before thus changes of $t$ task failure can not be attributed to changes in breathing pattern.

Further, one could argue that a reduction of $t$ task failure at $t 5$ might be a consequence of pre-existing fatigue of the respiratory muscles due to breathing against resistance at $t 0$ as LAGHi et al. [18] and Travaline et al. [19] showed diaphragmatic fatigue to last for at least $24 \mathrm{~h}$ after subjects breathed against inspiratory resistive loads at $60 \%$ of maximal transdiaphragmatic pressure $(P \mathrm{di}, \max )$ for $33 \mathrm{~min}$ and at $80 \% P$ di,max for $25 \mathrm{~min}$. However, those loaded breathing tasks were substantially longer than the resistive breathing test of the present study (average $6 \mathrm{~min}$ ). In a preliminary test, respiratory muscle performance of the subjects was assessed in two subsequent constant-load resistive breathing tests until exhaustion with a 15 min rest in between. In this setup, the second breathing test was of similar duration as the first one which suggests that the reduced time to exhaustion at $t 5$ was not the result of the pre-cycling resistive breathing test at $t 0$. In addition, if respiratory muscles were fatigued by the first test, a change in breathing pattern, similar to results of MADOR and ACEVEDo [20] would have been expected and possibly a change in endurance time during cycling [21]. This was not the case (table 3 ): breathing pattern as well as cycling times were similar during the three cycling endurance tests performed which also indicates that the reduced respiratory performance at $t 5$ is unlikely to be the result of a different load on respiratory muscles during cycling in the Task failure-series compared to the SVCor Spiro-series.

The possibility that central drive to respiratory muscles was reduced at $t 5$ (Task failure-series) to protect respiratory muscles from fatigue during constant-load resistive breathing also needs to be considered. It may in fact be possible that subjects always stopped the resistive breathing test because of a "protective" reduction of central drive. In any case, this would suggest, it is believed, that respiratory muscles were more prone to fatigue or slightly fatigued at $t 5$, thus "protection" was necessary earlier than during the test at $t 0$. Also, this potential reduction of central drive would have occurred later during resistive breathing tests at $t 40$ and $t 75$, which could indicate that respiratory muscles recovered in-between.

Lastly, one could argue that lactic acidosis, known to impair muscle contractility, was increased during constantload resistive breathing at $t 5$ compared to $t 0$ as a result of 
prior cycling. Indeed, blood lactate concentration was significantly increased at $t 5$ but similar increases were observed at $t 5$ of the SVC- and Spiro-series; in fact, blood lactate concentrations at $t 5$ were not significantly different between the three test series. Thus it is suggested that all respiratory tests performed at $t 5$ should have been affected similarly if blood lactate concentrations of $7 \mathrm{mmol} \cdot \mathrm{L}^{-1}$ would have influenced respiratory muscle performance at this time.

The question remains as to why respiratory muscle performance was only reduced during the constant-load resistive breathing test. It is obvious that the different respiratory performance measurements tested for different properties of respiratory muscles: the maximal isometric force $(P \max )$ and the ability to perform fast contractions with little development of force over a short period of time (FEV1, PEF, MVV) and over a longer period of time (SVC) remained unaffected by prior exercise while the ability to produce a high force with slow movement over an extended period of time was diminished. Why only this last type of respiratory performance was impaired after exercise remains to be addressed in further studies.

The aim of the present study was to apply different noninvasive techniques, commonly used in the literature to detect respiratory muscle fatigue $[5,8,16]$, for the measurement of possible impairments of respiratory muscle performance after a type of exercise which was previously shown to induce diaphragmatic fatigue [2-4]. Also, the authors wanted to compare measures of reduced performance with the published data on reductions in Pdi,tw. In the present study, respiratory muscle performance during constant-load resistive breathing was reduced by $43 \%$ at $5 \mathrm{~min}$ after the end of exercise $\left(87 \% V^{\prime} \mathrm{O}_{2}\right.$, max, corresponding to $78 \% W \max$ ) while the decrease of $P$ di,tw was $17 \%$ at $10 \mathrm{~min}$ after cycling $\left(80 \% W_{\max }\right)[2]$ and ranged from $8-32 \%$ immediately after cycling $(85-95 \%$ $\left.V^{\prime} \mathrm{O}_{2}, \max \right)$ [4]. Since the exercise workload of the present study corresponded well to the workloads in the above mentioned studies, diaphragmatic fatigue was also likely to be present in the current subjects. If so, a possible explanation for the differences in the decrease of respiratory performance and the decrease of $P$ di,tw might then be that during constant-load resistive breathing not only the diaphragm but also inspiratory ribcage muscle performance was tested. This can be inferred from a study suggesting that breathing against a threshold load preferentially fatigues ribcage muscles rather than the diaphragm [22]. In fact, McKenzie et al. [23] were unable to detect diaphragmatic fatigue in their subjects at the point of task failure after breathing against resistive loads. It is possible that, during cycling, rib cage muscles fatigue to a similar or larger extent than the diaphragm: this assumption is supported by JoHnson et al. [4] who have shown that the relative contribution of the diaphragm to total respiratory motor output was progressively reduced with exercise. If ribcage muscles do fatigue during exercise, a task testing ribcage muscle performance as well as diaphragmatic performance would be affected to a larger extent by prior exercise than a test measuring diaphragmatic fatigue only.

While $t$ task failure at $t 5$ was significantly reduced compared to $t 0$, the differences were no longer significant at $t 40$ (although 8/12 subjects had not yet attained control values), and at $t 75$ baseline levels were reached. This pattern of recovery corresponds to the pattern of $P$ di,tw recovery measured in previous studies where $P$ di,tw returned to baseline values after $60 \mathrm{~min} \mathrm{[2]} \mathrm{and} 70 \mathrm{~min} \mathrm{[4]} \mathrm{but} \mathrm{the}$ same pattern of recovery was also shown for ribcage muscles. SimiLOWSKi et al. [24] found that ribcage muscles fully recovered within $60 \mathrm{~min}$ of the fatiguing exercise. Since the time course of recovery was similar in the present study, it might be possible that constant-load resistive breathing does test for global inspiratory muscle fatigue.

In summary, the constant-load resistive breathing test to task failure applied in the present study seems to be a useful tool for detecting a reduction in respiratory muscle performance capacity due to prior respiratory muscle work. This test could be useful in assessing respiratory muscle performance of patients suffering from chronic obstructive pulmonary disease and cystic fibrosis since these patients also benefit from inspiratory resistive or threshold training [25]. The constant-load resistive breathing test could then be used to follow progress or reduction in respiratory muscle performance over time.

In conclusion, measures of vital capacity, forced vital capacity, forced expiratory volume in one second, peak expiratory flow, maximal voluntary ventilation in $15 \mathrm{sec}-$ onds, maximal inspiratory and expiratory mouth pressures or sustained ventilatory capacity were not affected by exhaustive cycling at $85 \%$ maximal oxygen uptake. Thus, it is concluded that constant-load resistive breathing to task failure is the only noninvasive respiratory muscle performance test evaluated in this study that shows a decrease in respiratory muscle performance after exhaustive endurance exercise.

Acknowledgements. The authors thank S.M. Kelly, for editorial comments on the manuscript.

\section{References}

1. NHLBI Workshop Summary. Respiratory muscle fatigue: report of the respiratory muscle fatigue workshop group. Am Rev Respir Dis 1990; 142: 474-480.

2. Mador MJ, Magalang UJ, Rodis A, Kufel TJ. Diaphragmatic fatigue after exercise in healthy human subjects. Am Rev Respir Dis 1993; 148: 1571-1575.

3. Babcock MA, Pegelow DF, McClaran SR, Suman OE, Dempsey JA. Contribution of diaphragmatic power output to exercise-induced diaphragm fatigue. $J$ Appl Physiol 1995; 78: 1710-1719.

4. Johnson BD, Babcock MA, Suman OE, Dempsey JA. Exercise-induced diaphragmatic fatigue in healthy humans. J Physiol (Lond) 1993; 460: 385-405.

5. Loke J, Mahler DA, Virgulto JA. Respiratory muscle fatigue after marathon running. J Appl Physiol 1982; 52: 821-824.

6. Hill NS, Jacoby C, Farber HW. Effect of an endurance triathlon on pulmonary function. Med Sci Sports Exerc 1991; 23: 1260-1264.

7. Chevrolet J-C, Tschopp J-M, Blanc Y, Rochat T, Junod AF. Alterations in inspiratory and leg muscle force and recovery pattern after a marathon. Med Sci Sports Exerc 1993; 25: 501-507.

8. McConnell AK, Caine MP, Sharpe GR. Inspiratory muscle fatigue following running to volitional fatigue: the influence of baseline strength. Int J Sports Med 1997; 18: $169-173$. 
9. Warren GL, Cureton KJ, Sparling PB. Does lung function limit performance in a 24-hour ultramarathon? Respir Physiol 1989; 78: 253-264.

10. Mahler DA, Loke J. Pulmonary dysfunction in ultramarathon runners. Yale J Biol Med 1981; 54: 243-248.

11. Mahler DA, Snyder P, Loke J. Pulmonary function in runners before and after a 20 kilometer road race. Conn Med 1980; 44: 549-552.

12. Maron MB, Hamilton LH, Maksud MG. Alterations in pulmonary function consequent to competitive marathon running. Med Sci Sports Exerc 1979; 11: 244-249.

13. Miles DS, Durbin RJ. Alterations in pulmonary function consequent to a 5-mile run. J Sports Med 1985; 25: 90 97.

14. Miles DS, Doerr CE, Schonfeld SA, Sinks DE, Gotshall RW. Changes in pulmonary diffusing capacity and closing volume after running a marathon. Respir Physiol 1983; 52: 349-359.

15. Nava S, Zanotti E, Rampulla C, Rossi A. Respiratory muscle fatigue does not limit exercise performance during moderate endurance run. J Sports Med Phys Fitness 1992; 32: 39-44.

16. Ker JA, Schultz CM. Respiratory muscle fatigue after an ultra-marathon measured as inspiratory task failure. Int $J$ Sports Med 1996; 17: 493-496.

17. Clanton TL, Dixon GF, Drake J, Gadek JE. Effects of breathing pattern on inspiratory muscle endurance in humans. J Appl Physiol 1985; 59: 1834-1841.
18. Laghi F, D'Alfonso N, Tobin MJ. Pattern of recovery from diaphragmatic fatigue over 24 hours. J Appl Physiol 1995; 79: 539-546.

19. Travaline JM, Sudarshan S, Criner GJ. Recovery of PdiTwitch following the induction of diaphragm fatigue in normal subjects. Am J Respir Crit Care Med 1997; 156: $1562-1566$.

20. Mador MJ, Acevedo FA. Effect of respiratory muscle fatigue on breathing pattern during incremental exercise. Am Rev Respir Dis 1991; 143: 462-468.

21. Mador MJ, Acevedo FA. Effect of respiratory muscle fatigue on subsequent exercise performance. $J$ Appl Physiol 1991; 70: 2059-2065.

22. Hershenson MB, Kikuchi Y, Tzelepis GE, McCool FD. Preferential fatigue of the rib cage muscles during inspiratory resistive loaded ventilation. $J$ Appl Physiol 1989; 66: 750-754.

23. McKenzie DK, Allen GM, Butler JE, Gandevia SC. Task failure with lack of diaphragm fatigue during inspiratory resistive loading in human subjects. J Appl Physiol 1997; 82: 2011-2019.

24. Similowski T, Straus C, Attali V, Duguet A, Derenne J-P. Cervical magnetic stimulation as a method to discriminate between diaphragm fatigue and rib cage muscle fatigue. $J$ Appl Physiol 1998; 84: 1692-1700.

25. Reid WD, Samrai B. Respiratory muscle training for patients with chronic obstructive pulmonary disease. Phys Ther 1995; 75: 996-1005. 